\title{
Avaliação do tempo de resposta eletromiográfica em atletas de voleibol e não atletas que sofreram entorse de tomozelo
}

\author{
Adriana Moré Pacheco ${ }^{1}$, Marco Aurélio Vaz² e Ivan Pacheco ${ }^{3}$
}

\section{RESUMO}

A proposta deste estudo foi examinar o tempo de resposta eletromiográfica dos músculos fibulares, na inversão repentina do pé, em tornozelos com presença de lesão e saudáveis. Três grupos foram testados, um de atletas normais (grupo 1), um de atletas com história recente de entorse de tornozelo (grupo 2) e o outro de não atletas com história recente de entorse de tornozelo (grupo 3). Para cada sujeito dos três grupos, ambos os tornozelos foram testados. Os sujeitos que sofreram entorse de tornozelo (grupos 2 e 3) não apresentavam sintomas de lesão durante os últimos dois meses antes do teste. Uma plataforma capaz de produzir uma inversão repentina lateral de $20^{\circ}$ do tomozelo no plano frontal simulava um evento de entorse de tomozelo. Eletrodos de eletromiografia de superfície foram colocados na pele sobre os músculos fibulares. Os tempos de resposta eletromiográfica dos músculos fibulares foram obtidos e comparados entre os grupos. Para o grupo 1, a média dos tempos de resposta eletromiográfica foi de $71 \mathrm{~ms}$ para a perna direita e 69ms para a perna esquerda. Para o grupo 2, a média dos tempos de resposta eletromiográfica foi de $72 \mathrm{~ms}$ para o tornozelo sem lesão e $74 \mathrm{~ms}$ para o tornozelo com a lesão. Para o grupo 3, a média dos tempos de resposta eletromiográfica foi de $72 \mathrm{~ms}$ para o tomozelo sem lesão e $73 \mathrm{~ms}$ para o tornozelo com a lesão. Os resultados indicaram que não houve diferença estatisticamente significante entre as pernas direita e esquerda no grupo 1 e entre os tornozelos sem lesão e com lesão dos grupos 2 e 3 para os músculos fibulares. Os achados do presente estudo sugerem que a resposta eletromiográfica dos músculos fibulares, durante o deslocamento angular repentino do tornozelo, não foi influenciada pela entorse de tomozelo.
1. Fisioterapeuta do Clube Grêmio Náutico União - POA/RS, Fisioterapeuta da Clínica SOS ESPORTE, Mestre em Ciências do Movimento Humano - UFRGS, Professora do Curso de Fisioterapia da PUCRS, Doutoranda em Ciências do Movimento Humano - UFRGS.

2. Pós-Doutorado Universidade de Calgary, Canadá, Doutor pela Universidade de Calgary, Canadá, Professor do Curso de Educação Física da UFRGS, Professor do Programa de Pós-Graduação em Ciências do Movimento Humano da ESEF/UFRGS.

3. Médico Ortopedista e Traumatologista do Esporte da Clínica SOS ESPORTES, M édico Ortopedista e Traumatologista do Esporte e Coordenador do Centro de Medicina e Reabilitação do Clube Grêmio Náutico União - POA/RS, Diretor médico da Federação Gaúcha de Futevôlei e Diretor médico da Federação Gaúcha de Futebol, Mestre em Ciências do Movimento Humano - UFRGS, Doutor em Ciências do Movimento Humano - UFRGS.

Recebido em 26/9/04. Versão final recebida em 15/6/05. Aceito em 17/7/05. Endereço para correspondência: Adriana Moré Pacheco, Rua Comendador Rheingantz, 362, apto. 601, B. Auxiliadora - Porto Alegre, RS. Email: adrimpacheco@terra.com.br

\begin{abstract}
Palavras-chave: Tempo de resposta eletromiográfica. Entorse. Tornozelo.
Keywords: $\quad$ Time for the electromyographic response. Sprain. Ankle.

Palabras-clave: Tiempo de respuesta eletromiográfica. Esguince. Tobillo.
\end{abstract}

\section{ABSTRACT}

Evaluation of the time for the electromyographic response in volleyball athletes and non-athletes who had ankle sprain

The purpose of this study was to examine the time for the electromyographic response of the fibular muscles in the sudden foot inversion in sprained and healthy ankles. Three groups of athletes were tested: one composed by healthy athletes (group 1), one group of athletes with recent history of ankle sprain (group 2), and another group composed by non-athletes with recent history of ankle sprain (group 3). For each individual from the three groups both ankles were tested. Individuals with ankle sprain (groups 2 and 3) were asymptomatic for the last two months prior to the test. A platform able to produce a sudden $20^{\circ}$ side inversion of the ankle in the frontal plane simulated an ankle sprain event. Surface electromyography electrodes were placed over the fibular muscles. Times for the electromyographic response of the fibular muscles were obtained and compared between groups. In group 1, the mean electromyographic response times were: $71 \mathrm{~ms}$ for the right leg, and $69 \mathrm{~ms}$ for the left leg. In group 2, the mean electromyographic response times were: 72 ms for non-sprained ankle, and 74 ms for sprained ankle. In group 3, the mean electromyographic response times were: $72 \mathrm{~ms}$ for non-sprained ankle and $73 \mathrm{~ms}$ for sprained ankle. Results indicated no statistically significant difference between the right and left legs in group 1, and between non-sprained and sprained ankle in groups 2 and 3 for the fibular muscles. The findings in the present study suggest that the time for the electromyographic response of the fibular muscles to sudden angular displacement of the ankle was not influenced by the ankle sprain.

\section{RESUMEN}

Evaluación del tiempo de respuesta electromiográfica en atletas de voleibol y no atletas que han sufrido esguince de tobillo

La propuesta de este estudio era examinar el tiempo de la respuesta electromiográfica de los musculos fibulares, en el esguince agudo del pie, en los tobillos con la presencia de lésion y saludables. Se probaron tres grupos, uno de atletas normales (grupo 1), uno de atletas con la reciente historia de esguince del tobillo (grupo 2) y el otro de ningún atleta con la reciente historia de esguince del tobillo (grupo 3). Para cada sujeto de los tres grupos, se probaron ambos tobillos. Los atletas que sufrieron el esguince del tobillo (se agruparon en 2 y 3) estos no presentaron los síntomas de la lesión durante los últimos dos meses antes de la prueba. Se usó una plataforma capable di producir una inversión súbita lateral de $20^{\circ}$ del tobillo en el plan delantero simulando, así, un evento de esguince del tobillo. Se pusieron electrodos de eletromiografia de la superficie en la piel en la región anexa de los músculos. Se 
obtuvieron registros eletromiográficos de la respuesta de las fibras de los músculos y se compararon, así, entre los grupos. Para el de grupo 1, el promedio de las veces de eletromiográfica de la respuesta fue de $71 \mathrm{~ms}$ por la piema correcta y $69 \mathrm{~ms}$ para la pierna izquierda. Para el de grupo 2, el promedio de las veces de eletromiográfica de la respuesta fue de 72 malo por el tobillo sin la lesión y 74 malo para el tobillo con la lesión. Para el de grupo 3, el promedio de las veces de eletromiográfica de la respuesta fue de 72 malo por el tobillo sin la lesión y 73 malo para el tobillo con la lesión. Los resultados indicaron que no había diferencia estatisticamente significante entre las piemas sanas y lo hallado en el grupo 1 y entre los tobillos sin la lesión y con la lesión de los grupos 2 y 3 para las fibras de los músculos. Los descubrimientos del estudio presente sugieren que la eletromiográfía de la respuesta de las fibras de los músculos, durante el desplazamiento angular aguso del tobillo, no es influenciada por el esguince del tobillo.

\section{INTRODUÇÃO}

A entorse é considerada a lesão de maior ocorrência na articulação do tomozelo(1). A alta incidência desse tipo de lesão se dá durante a prática tanto de esportes de contato ${ }^{(2,3)}$, como esportes em que não há contato entre os atletas, como é o caso do voleiboll(1,4-6). Tal fato se deve, principalmente, aos gestos esportivos que são executados nestes esportes, sendo os saltos e corridas e as quedas ao solo, após um salto, os principais responsáveis pelas lesões nos tomozelos $(21 \% \text { a } 25 \%)^{(3,7)}$. As entorses correspondem a $75 \%$ dessas lesões e o mecanismo por inversão chega a $85 \%$ ou $90 \%{ }^{(3,5,8)}$. Esta lesão caracteriza-se pelo estiramento e/ou ruptura de vários tecidos, rupturas ligamentares incompletas ou completas nos graus 2 e 3, respectivamente, afrouxamento capsular e instabilidade articular(9-12). As "deformações" produzidas por um estiramento excessivo dos tecidos ocorrem no compartimento lateral da perna, atingindo, principalmente, os músculos fibulares curto e longo. Com esse estiramento, podem ocorrer situações como alteração da capacidade proprioceptiva pela lesão e pela instabilidade articular(13-16).

As entorses podem ser classificadas de acordo com a intensidade do trauma em: a) Grau I ou leve - a integridade de quase todas as fibras ligamentares é mantida. Encontra-se pequena reação vasomotora, caracterizada, geralmente, pelo edema. Há presença de dor leve na fase aguda. Existe pronto restabelecimento do apoio e da marcha. b) Grau II ou moderado - há presença de hematoma e edema de maior dimensão, devido a uma maior lesão vascular. Pelo teste de gaveta anterior, verifica-se pequena instabilidade quando a articulação é submetida ao exame sob estresse. Existe presença de quadro álgico e inflamatório maior que no grau I, e, desta maneira, o apoio e a marcha são dificultados, retomando somente após a regressão desses sintomas. Neste grau ocorre ruptura parcial dos ligamentos. c) Grau III ou grave apresenta-se com dor intensa, há grande área de ruptura de vasos mostrando edema importante, hematoma de grande extensão e, ainda, tumefação na articulação do tornozelo. Ocorre instabilidade radiológica por estresse de grande abertura, e pode haver avulsões ósseas. No teste de gaveta anterior, também é possível a verificação de grande instabilidade. A ruptura das estruturas capsuloligamentares é completa, e isso se comprova por artrografia devido ao extravasamento de líquido para as regiões onde, normalmente, ele não deve ser encontrado. Neste grau, o tratamento é geralmente cirúrgico ${ }^{(9-12)}$.

Uma das formas de detectar a redução na capacidade proprioceptiva devido à entorse de tomozelo é através da deteminação do tempo de resposta eletromiográfica dos músculos eversores do pé, através da eletromiografia de superfície ${ }^{(17-21)}$. Sendo assim, o tempo de resposta eletromiográfica, se define como o valor da diferença que se pode obter entre o estímulo do músculo, que pode ser dado através de um movimento repentino para acionar a distensão da musculatura, até a reação deste músculo a este estímulo. Na entorse do tornozelo por inversão, a musculatura que deve ser acionada é a dos eversores do compartimento lateral da perna, através da simulação de um mecanismo de entorse. Esta resposta eletromiográfica é medida em milissegundos e pode ser obtida pela eletromiografia de superfície.

Evidências nesse sentido foram apresentadas em estudos que avaliaram a resposta postural de sujeitos saudáveis e pacientes com história recente de entorse de tomozelo a partir de mudanças em uma superfície de apoio dos pés, criadas durante um balanço no plano frontal. Os resultados confirmaram que a redução da capacidade proprioceptiva, após uma entorse que afetou a articulação do tomozelo, resultou em um atraso no tempo de resposta eletromiográfica dos músculos fibular longo e tibial anterior, registrado pela eletromiografia(13). No entanto, essa idéia tem sido disputada por outros autores que não encontraram qualquer alteração no tempo de resposta eletromiográfica dos músculos eversores do pé entre indivíduos saudáveis e indivíduos com história de entorse de tomozelo(8,20,22). Tendo em vista o aumento do número de praticantes de voleibol no Brasil e no mundo e a alta incidência de entorse de tornozelo nesse esporte $(1,4,6,23)$, a resolução das controvérsias em relação ao uso do tempo de resposta eletromiográfica, como forma de investigação da redução da capacidade proprioceptiva de atletas, seria de grande valia no sentido de auxiliar médicos e fisioterapeutas na determinação do momento em que um atleta pode retornar às atividades normais de treinamento. No entanto, não foi encontrado na literatura nenhum estudo sistemático que tenha avaliado a resposta elétrica dos músculos de atletas de voleibol saudáveis e com história recente de entorse de tornozelo.

Portanto, com base na investigação da existência ou não de um atraso no tempo de resposta eletromiográfica, a partir da utilização de uma plataforma com $20^{\circ}$ de queda, a qual simula os movimentos de inversão da articulação do tomozelo e a eletromiografia de superfície para registrar a atividade elétrica muscular, o objetivo do presente estudo foi comparar os tempos de resposta eletromiográfica dos músculos eversores (fibulares curto e longo) do pé de atletas com tomozelos saudáveis e atletas e não atletas com história recente de entorse em inversão do tornozelo.

\section{MATERIAL E MÉTODOS}

A amostra consistiu de 16 atletas profissionais de voleibol com faixa etária compreendida entre 14 e 25 anos de idade (média de 17,5 anos, com desvio padrão de 2,12 e a moda =16) e 15 indivíduos não atletas, de ambos os sexos, com faixa etária compreendida entre 19 e 40 anos de idade (média de 25,06 anos, com desvio padrão de 5,82 e a moda $=22$ ). A amostragem deste estudo foi feita de forma intencional. Três grupos foram formados para a coleta de dados. O grupo 1 (grupo controle) foi composto por atletas praticantes de voleibol nos últimos três anos, com freqüência de treinamento de cinco vezes por semana e três horas por dia, considerados profissionais, e que apresentavam os tomozelos saudáveis. O grupo 2 foi composto também por atletas de voleibol com as mesmas características do grupo 1, porém apresentavam história recente de entorse de tornozelo com classificação grau II(9-12) unilateral por inversão do pé. O grupo 3 foi composto por indivíduos não atletas, também com história recente de entorse de tornozelo por inversão, com classificação grau II(9-12). Os sujeitos que sofreram entorse de tomozelo (grupos 2 e 3) não apresentavam sintomas de lesão durante os últimos dois meses antes do teste. Portanto os critérios de inclusão para o estudo eram atletas de voleibol com prática de três anos da modalidade, com entorse de tornozelo classificado como grau $\|^{(9-12)}$, e outros com tornozelos sem lesão e indivíduos não atletas com tomozelos saudáveis. Os critérios de exclusão do estudo compreendiam os atletas de voleibol com prática da modalidade inferior a três anos, que apre- 
sentavam lesões nos quadris e joelhos ou diagnóstico de outra lesão no tornozelo que não a entorse de $2 \circ$ grau. Também eram excluídos os sujeitos não atletas com lesões nos quadris e joeIhos ou diagnóstico de outra lesão no tomozelo que não a entorse de $2 \circ$ grau.

\section{Procedimento para a coleta dos dados}

Foi utilizado como história recente de entorse de tornozelo a fase compreendida entre a quarta e a décima semanas após a lesão, época em que todos os sujeitos do estudo foram analisados. Os indivíduos do grupo 2 foram avaliados com média do tempo de entorse de 6,8 semanas (desvio padrão de 2,70) e o grupo 3 com média do tempo de entorse de 5,8 semanas (desvio padrão de 2,20). Este período estava relacionado ao fato de que as fibras de colágeno já estariam suportando cargas quase normais, sendo esta a fase em que os sujeitos são liberados para retornarem à prática esportiva(24,25).

Para o diagnóstico de entorse de tornozelo de segundo grau, era realizado um exame físico, através do teste de gaveta anterior, sempre pelo mesmo médico traumatologista e pela classificação de acordo com a intensidade do trauma apresentada na literatura, em grau I ou leve, grau II ou moderado e grau III ou grave(9-12). A partir do diagnóstico, os atletas e os pacientes eram tratados com imobilizador do tipo Air Cast, todos pelo mesmo médico traumatologista, e eram encaminhados para tratamento fisioterápico. Quando se encontravam no final da quarta semana de tratamento estes eram encaminhados ao Laboratório do Exercício (LAPEX) da Universidade Federal do Rio Grande do Sul (UFRGS) para início das coletas dos dados. Neste momento, os sujeitos não apresentavam nenhum sinal de dor nem queixas de instabilidade.

Um eletromiógrafo de oito canais (Bortec Electronics Incorporation, Canadá) foi utilizado para a aquisição dos sinais eletromiográficos (EMG). Eletrodos adesivos descartáveis de superfície, em configuração bipolar (1 centímetro de diâmetro cada), foram posicionados no ventre dos músculos fibulares (da perna direita e esquerda) no sentido de seu eixo longitudinal (1/3 abaixo da cabeça da fíbula). A distância entre os eletrodos foi de aproximadamente três centímetros. Eletrodo de aterramento, também adesivo e descartável, foi posicionado na tuberosidade anterior da tíbia (esquerda), paralelo ao posicionamento dos eletrodos dos músculos fibulares. A pele sob os eletrodos foi preparada para a coleta dos sinais eletromiográficos reduzindo-se a impedância elétrica pela raspagem dos pêlos, com uma gilete descartável e pela abrasão da pele, com algodão embebido em álcool, a fim de remover as células mortas e a oleosidade no local do posicionamento dos eletrodos ${ }^{(26)}$. A seguir os eletrodos eram fixados na pele e era aplicada uma leve pressão sobre eles para aumentar o contato entre o gel do eletrodo e a pele(27). A impedância entre os eletrodos foi medida através de um voltímetro e mantida abaixo de $5 \mathrm{KOhms.} \mathrm{As} \mathrm{duas}$ pernas foram preparadas igualmente, pois a que apresentava o tornozelo saudável serviu de controle.

Os sinais eletromiográficos foram coletados juntamente com o sinal da plataforma (sincronismo). Esses sinais foram amostrados a uma freqüência de $4.000 \mathrm{~Hz}$. O sinal eletromiográfico foi filtrado com filtro ideal de freqüência mínima $20 \mathrm{~Hz}$ e máxima de $700 \mathrm{~Hz}$.

Uma plataforma de inversão foi desenvolvida pelo Laboratório de Medições Mecânicas da Escola de Engenharia da UFRGS em Porto Alegre/RS. Essa plataforma ou prancha permitia a inversão do pé (no plano frontal) e simulava um movimento de inversão subtalar de $20^{\circ}$. Essa plataforma era semelhante à utilizada por Karlsson et al. (1992)(19). Um sistema de sincronismo manual foi instalado na plataforma, a fim de gerar um sinal elétrico que indicasse o início do movimento de inversão do tornozelo. Esse sistema era individual para cada lado da plataforma e serviu para sincronizar os eventos de inversão do tomozelo com a ativação elétrica do músculo. Esse sinal era acionado, pelo pesquisador, por meio de cordas, que eram puxadas independentes para cada lado da plataforma (figura 1). Assim, quando um dos lados da plataforma caísse, pelo puxar da corda, o sincronismo então era desligado gerando um sinal no monitor do computador, juntamente com o sinal elétrico do músculo proveniente da eletromiografia. A diferença entre estes dois sinais correspondia ao tempo de resposta eletromiográfica do músculo estudado.

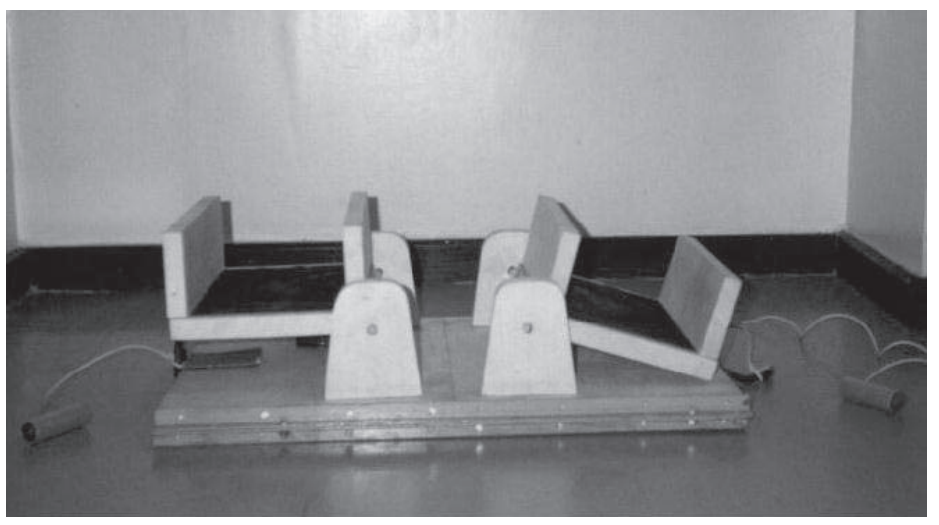

Fig. 1 - Plataforma de inversão: demonstra o deslocamento angular de $20^{\circ}$ da tábua do lado direito, ao qual eram submetidos os indivíduos durante o experimento. A tábua do lado esquerdo demonstra a posição em que o tornozelo esquerdo do indivíduo permanecia, enquanto ocorria o deslocamento do lado direito.

O posicionamento dos sujeitos na plataforma adotou um procedimento padrão (figura 2), em que estes permaneciam de olhos abertos ou olhos fechados ${ }^{(18)}$, executando um protocolo que consistiu de doze movimentos de inversão produzidos de forma aleatória nos dois tomozelos, sendo seis inversões com o indivíduo de olhos abertos e seis com o indivíduo de olhos fechados, conforme protocolo aleatório. Foram utilizados três movimentos para cada lado, com a finalidade de se evitar um possível treinamento da tarefa. Foi adotado um cuidado para que todas as inversões fossem repentinas e inesperadas. Para a utilização desse fator surpresa, mecanismos como conversas, entre o pesquisador e o auxiliar da pesquisa, foram adotadas para que o indivíduo se distraísse e não soubesse qual seria o momento da inversão do tornozelo e de qual lado aconteceria. Nenhum sinal de dor foi mencionado pelos sujeitos durante os testes. Os sinais eletromiográficos foram coletados juntamente com o sinal da plataforma (sincronismo).

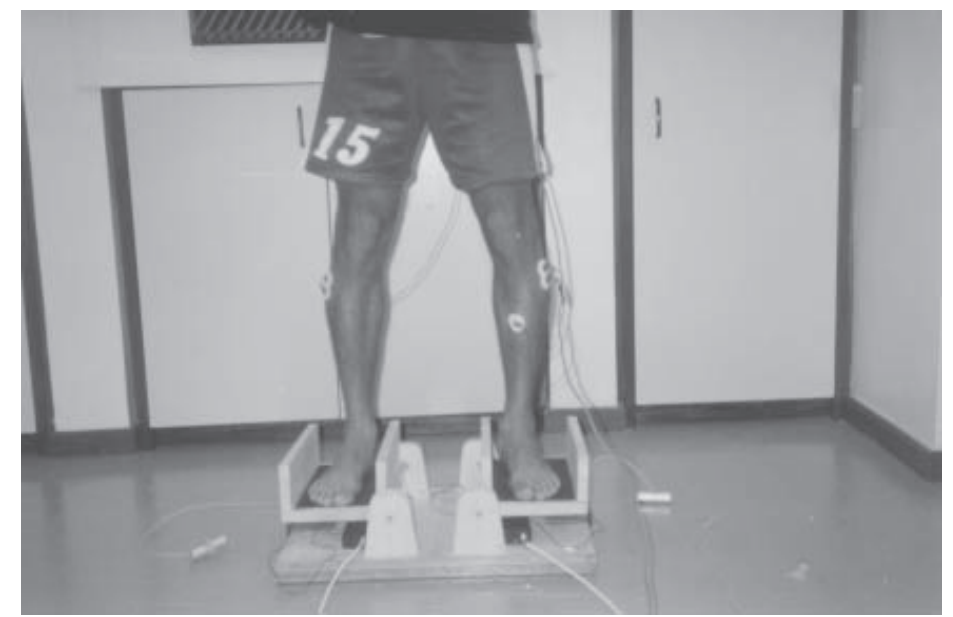

Fig. 2 - Vista anterior do sujeito na plataforma

Todos os procedimentos do estudo foram aprovados pelo Comitê de Ética em Pesquisa do Hospital de Clínicas de Porto Alegre/RS, sob o número 01-074 em 28 de março de 2001, e aprovados pelo consentimento informado de cada indivíduo. 


\section{Análise estatística}

O teste t pareado foi utilizado em cada grupo para comparar os tempos de resposta eletromiográfica entre os indivíduos com os olhos abertos e com os olhos fechados para o tornozelo direito e para o tomozelo esquerdo e comparação entre os tornozelos direito e esquerdo (grupo 1) e entre os tornozelos sem entorse e com entorse (grupos 2 e 3), em relação aos tempos de resposta eletromiográfica.

O nível de significância usado foi de $p<0,05$ para todas as análises.

\section{RESULTADOS}

Nos gráficos apresentados a seguir, foi utilizado como legenda para o grupo 1, tomozelo direito e tornozelo esquerdo, pois tratase da análise de um grupo sem lesão, portanto com os tornozelos saudáveis. Para os grupos 2 e 3, foram utilizados como legenda os termos sem lesão e com lesão, pois são grupos de indivíduos com entorse de tornozelo de $2^{\circ} \mathrm{grau}$.

\section{Tempo de resposta eletromiográfica}

Os resultados referentes ao tempo de resposta eletromiográfica obtidos para os três grupos podem ser visualizados na figura 3 . Não foi encontrada diferença significativa entre os tornozelos direito e esquerdo no grupo de atletas saudáveis (grupo 1), e entre os tomozelos sem lesão e o acometido pela entorse nos grupos 2 e 3 .

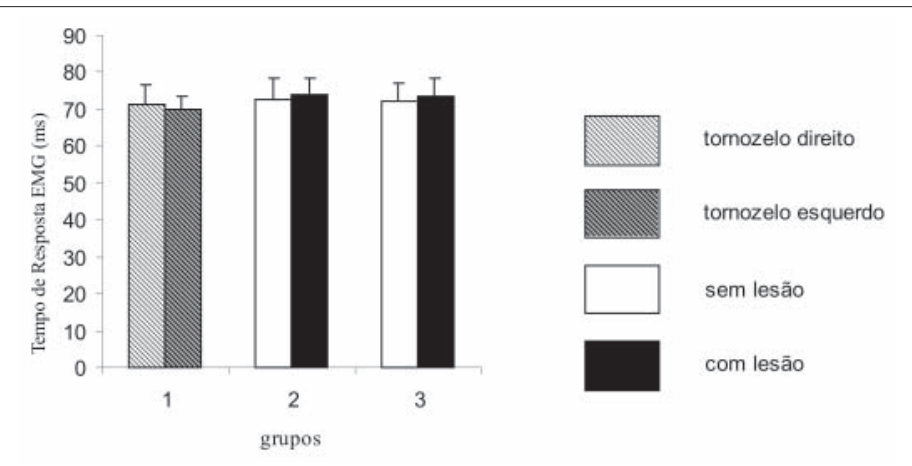

Fig. 3 - Tempo de resposta eletromiográfica (EMG) obtido para os três grupos da amostra

Também não foi encontrada diferença significativa para os resultados do tempo de resposta eletromiográfica nas situações olhos abertos comparados com olhos fechados em nenhum dos três grupos (figuras 4 e 5 ).

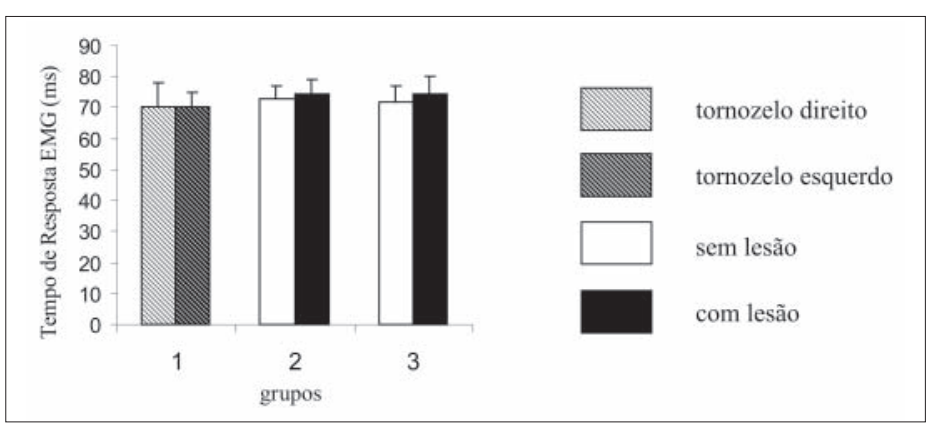

Fig. 4 - Tempo de resposta eletromiográfica (EMG) na situação com os olhos abertos

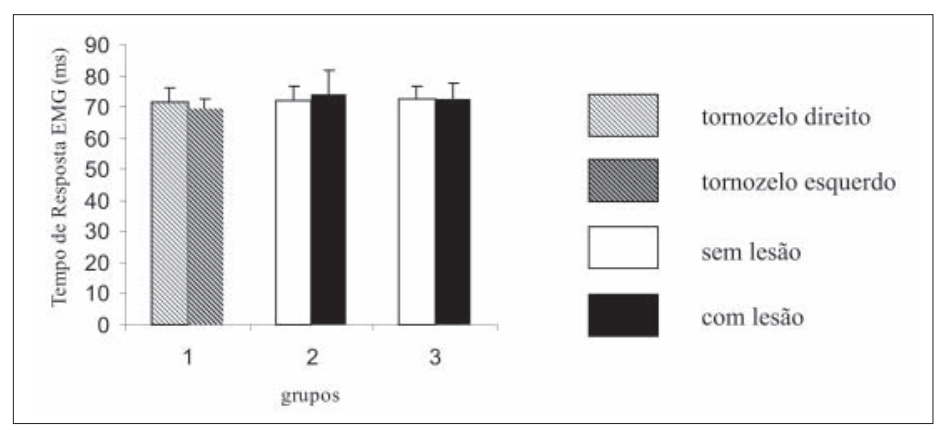

Fig. 5 - Tempo de resposta eletromiográfica (EMG) na situação com os olhos fechados

\section{DISCUSSÃO}

A entorse é considerada a lesão de maior ocorrência na articulação do tornozelo(1), principalmente em esportes envolvendo saltos e quedas, como é o caso do voleibol(1,4-6). Nesse tipo de lesão, a ruptura parcial ou total de diversos tecidos altera (ou diminui) a capacidade proprioceptiva de um atleta(13-16). Essa redução na propriocepção de um indivíduo parece ser responsável pela reincidência desse tipo de lesão ${ }^{(4,6,8,2,23,28,29)}$. A determinação do tempo de resposta eletromiográfica tem sido sugerida como uma das formas de detectar essa redução na capacidade proprioceptiva de um indivíduo acometido por entorse de tomozelo ${ }^{(18,19)}$. Essa redução na capacidade proprioceptiva deveria se manifestar como um aumento no tempo de resposta eletromiográfica dos músculos do segmento acometido pela lesão, quando comparado com o dos músculos do segmento saudável(19,21,30).

A revisão da literatura revelou que ainda existem controvérsias sobre o assunto, uma vez que alguns autores apresentaram evidências da inexistência de alteração no tempo de resposta eletromiográfica(3,8,13,20), enquanto outros trabalhos demonstram existir um atraso no tempo de resposta eletromiográfica ${ }^{(19,21,30)}$. Em vista das controvérsias que a literatura apresenta em relação aos tempos de resposta eletromiográfica, a proposta deste estudo foi comparar esses tempos de resposta elétrica dos músculos eversores (fibulares curto e longo) do pé de atletas com tornozelos saudáveis (grupo 1 ) e atletas e não atletas com história recente de entorse em inversão do tornozelo (grupos 2 e 3). Assumindo-se que o tempo de resposta eletromiográfica seria alterado devido à entorse de tornozelo, foram formuladas três hipóteses. A hipótese 1 era de que atletas profissionais de voleibol com tornozelos saudáveis (G.1) apresentariam um tempo de resposta eletromiográfica menor que os atletas profissionais de voleibol com história recente de entorse de tomozelo (G.2). Essa hipótese não se confimou, tendo em vista que os resultados para o grupo 1 e grupo 2 não apresentaram diferenças estatísticas. Na hipótese 2, esperava-se que atletas profissionais de voleibol com história recente de entorse de tornozelo (G.2) apresentassem um tempo de resposta eletromiográfica menor que indivíduos não atletas com história recente de entorse de tomozelo (G.3), tendo em vista as adaptações decorrentes de longo período de treinamento. Os resultados para esses grupos também não apresentaram diferenças estatísticas, portanto essa hipótese também não foi confirmada. $\mathrm{Na}$ hipótese 3 , atletas profissionais de voleibol com tomozelos saudáveis (G.1) deveriam apresentar um tempo de resposta eletromiográfica menor que indivíduos não atletas com história recente de entorse de tornozelo (G.3). Os resultados também não confirmaram essa hipótese, tendo em vista que também não foram encontradas diferenças estatísticas entre esses dois grupos.

Esses resultados corroboram achados da literatura, que vão contra a idéia de Freeman et al. (1965)(28), quando a instabilidade mecânica determinaria uma instabilidade funcional do tornozelo, acarretando uma incoordenação motora (ou aumento do tempo 
de resposta eletromiográfica) pela diminuição de estímulos dos mecanorreceptores como resultado da lesão ligamentar e/ou da cápsula articular, porém não confirmado nesses estudos $(3,8,13,20)$. Uma possível explicação para outros estudos que apresentarem alteração no tempo de resposta eletromiográfica pode estar relacionado com o ângulo de inversão produzido pelas plataformas utilizadas nesses estudos, o qual foi de $30^{\circ(19,21,30)}$. No caso do presente estudo, e dos demais que não encontraram diferenças significativas (3,8,22), o ângulo de inversão utilizado foi de apenas $20^{\circ}$, pois se acreditava que era um ângulo de inversão articular seguro para a utilização em indivíduos com lesão recente. Nesse sentido, este ângulo parece não ser suficientemente grande para revelar as alterações na capacidade proprioceptiva de tornozelos acometidos por entorse. A única exceção a esse caso foi um estudo que utilizaram um ângulo de $35^{\circ}$ (ou seja, o maior ângulo utilizado em todos os estudos), sem, no entanto, encontrarem diferenças significativas para os tempos de resposta eletromiográfica(20). Não fica claro o motivo dessa diferença em relação aos outros estudos que utilizaram um ângulo semelhante de movimento na plataforma de inversão.

Outro fato importante está relacionado ao tratamento fisioterápico, o qual provavelmente poderia ter apresentado influência nos estudos. O tratamento de fisioterapia, que serve para reabilitar a articulação lesada (no caso desses estudos, à articulação do tornozelo), não foi mencionado pelos autores que encontraram diferenças significativas como fator de reabilitação das entorses $(19,21,30)$. Como esses autores estavam trabalhando com tempos de histórico de entorses de tomozelo superiores a seis meses, isso nos leva a acreditar que a falta do tratamento fisioterápico tenha prejudicado a recuperação dos tecidos lesados concordando com estudos que relatam que o déficit proprioceptivo pode ser encontrado até dois anos após a lesão e, assim, o atraso no tempo de resposta eletromiográfica deverá permanecer ${ }^{(17)}$ (tabela 1).

\section{TABELA 1}

Resumo da metodologia encontrados na literatura

\begin{tabular}{llccc}
\hline \multicolumn{1}{c}{ Autor(es) } & EMG & Plataforma & $\begin{array}{c}\text { Tempo } \\
\text { de entorse }\end{array}$ & Fisioterapia \\
Isakov et al. (1986) & superfície & $20^{\circ}$ & 2 meses & Não \\
Konradsen e Ravn (1990) & superfície & $30^{\circ}$ & - & Não \\
Karlsson et al. (1992) & superfície & $30^{\circ}$ & 6 meses & Não \\
J ohnson e J ohnson (1993) & superfície & $35^{\circ}$ & 3 meses & Sim \\
Löfvenberg et al. (1995) & superfície & $30^{\circ}$ & 12 meses & Não \\
Ebig et al. (1997) & superfície & $20^{\circ}$ & 2 meses & Sim \\
Sheth et al. (1997) & inserção & $20^{\circ}$ & - & Sim \\
Presente estudo (2001) & superfície & $20^{\circ}$ & 4 a 10 semanas & Sim \\
\hline
\end{tabular}

Outro dado interessante, relacionado a algumas pesquisas, é que os resultados desses estudos parecem não fazer sentido, uma vez que os valores encontrados, para os grupos de tomozelos sem instabilidade e de tornozelos com instabilidade, parecem estar invertidos, ou seja, os valores maiores de tempo de resposta eletromiográfica foram encontrados para os grupos sem instabilidade articular do tornozelo (tornozelos saudáveis), enquanto os valores menores de tempo de resposta eletromiográfica foram para os tornozelos com lesão(3,8,20,22). No entanto, se a comparação for feita com os estudos que apresentaram uma diferença estatística, os resultados desses autores parecem apresentar uma certa lógi$\mathrm{ca}$, uma vez que os valores menores de tempos de resposta eletromiográfica foram para os tornozelos sem lesão e os maiores para os tornozelos com a presença de entorse $\mathrm{e}^{(19,21,30)}$.

\section{CONCLUSÃO}

Os achados do presente estudo sugerem que os tempos de resposta eletromiográfica para os músculos fibulares não são in- fluenciados pela entorse de tomozelo. Acredita-se que estes resultados foram influenciados pela angulação da plataforma, inferior a $30^{\circ}$, conforme se observa na discussão do artigo. Sugeremse estudos com plataformas de ângulos iguais a $30^{\circ}$ conforme achados da literatura que contemplaram seus objetivos de análise de atraso no tempo de resposta eletromiográfica.

\section{AGRADECIMENTOS}

Aos nossos familiares. Nossos pais, esposo, esposa e filha(s).

Todos os autores declararam não haver qualquer potencial conflito de interesses referente a este artigo.

\section{REFERÊNCIAS}

1. Carazzato J G, Campos LAN, Carazzato SG. Incidência de lesões traumáticas em atletas competitivos de dez tipos de modalidades esportivas. Rev Bras Ortop 1992;27:745-58

2. Derscheid GL, Brown WC. Rehabilitation of the ankle. Clin Sports Med 1985;4: 527-44.

3. Sheth $P, Y u$ B, Laskowski ER, An KN. Ankle disk training influences reaction times of selected muscles in a simulated ankle sprain. Am J Sports Med 1997;25:53843.

4. Schafle MD. Common injuries in volleyball. Treatment, prevention and rehabilitation. Sports Med 1993;16:126-9.

5. Ferretti A, De Carli A, Papandrea P. Volleyball injuries - A colour atlas of volleyball traumatology. Lausanne, Suisse: Federation Internationale de Volleyball, 1994; 27-41.

6. Bahr R, Bahr IA. Incidence of acute volleyball injuries: a prospective cohort study of injuries mechanisms and risk factors. Scand J Med Sci Sports 1997;7:166-71.

7. Holmer P, Sondergaard L, Konradsen L, Nielsen PT, J orgensen LN. Epidemiology of sprains in the lateral ankle and foot. Foot Ankle 1994;15:72-4.

8. Ebig M, Lephart SM, Burdett RG, Miller MC, Pincivero DM. The effect of sudden inversion stress on EMG activity of the peroneal and tibialis anterior muscles in the chronically unstable ankle. J Orthop Sports Phys Ther 1997;26:73-7.

9. O'Donoghue DH. Treatment of injuries to athletes. Philadelphia: W.B. Saunders, 1970.

10. Balduini FC, Tetzlaff J . Historical perspectives on injuries of the ligaments of the ankle. Clin Sports Med 1982;1:3-12.

11. Balduini FC, Vegso TJ, Torg J T, Torg E. Management and rehabilitation of ligamentous injuries to the ankle. Sports Med 1987;4:364-80.

12. Renström, Per AFH, Lynch SA. Lesões ligamentares do tornozelo. Rev Bras Med Esporte 1999;5:13-23.

13. Brunt D, Andersen J C, Huntsman B, Reinhert LB, Thorell AC, Sterling J C. Postural responses to lateral perturbation in healthy subjects and ankle sprain patients. Med Sci Sports Exerc 1992;24:171-6.

14. J erosch J , Prymka M. Proprioception and joint stability. Knee Surg Sports Traumatol Arthrosc 1996;4:171-9.

15. Konradsen L, Olesen S, Hansen HM. Ankle sensoriomotor control and eversion strength after acute ankle inversion injuries. Am J Sports Med 1998;26:72-7.

16. Watson AW. Ankle sprains in players of the field-game Gaelic football and hurling. J Sports Med Phys Fitness 1999;39:66-70.

17. Nitz AJ , Dobner J J Kersey D. Nerve injury and grades II and III ankle sprains. Am J Sports Med 1985;13:177-2.

18. Taimela S, Österman K, Kunjale U. M otor hability and personality with reference to soccer injuries. J Sports Med Phys Fitness 1990;30:194-01.

19. Karlsson J, Peterson L, Andreasson G, Högfors C. The unstable ankle: a combined EMG and biomechanical modeling study. Int J Sports Biomech 1992;8: 129-44.

20. J ohnson $M B$, J ohnson CL. Electromyographic response of peroneal muscles in surgical and nonsurgical injured ankles during sudden inversion. J Orthop Sports Phys Ther 1993;18:497-01.

21. Löfvenberg R, Kärrholm J , Sundelin G, Ahlgren O. Prolonged reaction time in patients with chronic lateral instability of the ankle. Am J Sports Med 1995;23: 414-7.

22. Isakov E, Mizrahi J , Solzi P, Susak Z, Lotem M. Response of the peroneal muscles to sudden inversion of the ankle during standing. Int J Sports Biomech 1986; 2:100-9.

23. Chan KM, Yuan Y, Li CK, Chien P, Tsang G. Sports causing most injuries in Hong Kong. BrJ Sports Med 1993;27:263-7. 
24. DeLee J C, Drez D J r. Orthopaedic sports medicine - Principle and practice. $2^{\text {nd }}$ ed. United States of America: W.B. Saunders Company, 1994;1705-67.

25. Hockembury RT, Sammarco GJ . Evaluation and treatment of ankle sprains. The Physician and Sports Med 2001;29:57-4.

26. Basmajian J V, De Luca CJ. Description and analysis of the EMG signal. Muscles alive: their functions revealed by electromyography. Baltimore, Williams and Wilkins: J ohn Butler, 1985;19-167.
27. Nigg BM, Herzog W. Biomechanics of the musculo-skeletal system. J ohn Wiley \& Sons, Toronto, 1994.

28. Freeman MAR. Instability of the foot after injuries to the lateral ligament of the ankle. J Bone J oint Surg Br 1965;47:669-7.

29. Freeman MAR, Dean MR, Hanham IWF. The etiology and prevention of functional instability of the foot. J Bone J oint Surg Br 1965;47:678-5.

30. Konradsen L, Ravn J B. Ankle instability caused by prolonged peroneal reaction time. Acta Orthop Scand 1990;61:388-90. 